\title{
The ESO nearby Abell cluster survey ${ }^{\star}$
}

\section{Morphological and spectral classification of galaxies ${ }^{\star}$}

\author{
T. Thomas and P. Katgert
}

Sterrewacht Leiden, PO Box 9513, 2300 RA Leiden, The Netherlands

e-mail: katgert@strw.leidenuniv.nl

Received 20 June 2005 / Accepted 14 September 2005

\section{ABSTRACT}

We determine the morphological types of 2295 galaxies from the ESO Nearby Abell Cluster Survey (ENACS) from CCD images obtained with the Dutch telescope on La Silla. A comparison with morphological types from the literature for 450 of our galaxies shows that the reliability of our classification is quite comparable to that of other classifiers. We recalibrate the ENACS spectral classification with the new morphological types, and find that early- and late-type galaxies can be distinguished from their spectra with $83 \%$ reliability. Ellipticals and S0 galaxies can hardly be distinguished on the basis of their spectra, but late spirals can be classified from the spectrum alone with more than $70 \%$ reliability. We derive pseudo-colors and linestrengths from the ENACS spectra for the galaxies of different morphological types. We consider the bright $\left(M_{R} \leq-20\right)$ and faint $\left(M_{R}>-20\right)$ subsets of the galaxies without emission lines (non-ELG) separately. We find a strong and significant correlation between the average color and the average strength of the metal absorption lines. The average metallicity decreases and the average color gets bluer towards later Hubble type. Also, the faint galaxies in each morphological class are bluer and less metal-rich than their brighter counterparts, which extends the well-established color-magnitude relation of early-type galaxies to (late) spirals. In view of these very strong global trends, the colors and metallicities of faint S0 galaxies and bright early spirals are remarkably similar. The bright early spirals may, on average, have somewhat stronger $\mathrm{H} \delta$ absorption than the other galaxies, which could be due to recent starformation.

The galaxies with emission lines (ELG) have a bluer spectral continuum than the non-ELG, and the amount of blueing hardly depends on morphological type. The fraction of ELG depends strongly on morphological type (varying from $4 \pm 1 \%$ for ellipticals to $59 \pm 4 \%$ for late spirals), but for each of the morphological types it varies very little with projected distance from the cluster center.

Key words. galaxies: clusters: general - galaxies: fundamental parameters - galaxies: statistics

\section{Introduction}

After the introduction by Hubble of his galaxy classification scheme $(1926,1936)$, various other schemes have been proposed, e.g. by Morgan (1958), de Vaucouleurs (1959), Sandage (1961) and van den Bergh (1960a,b, 1976). Although the details of those schemes differ, they all share Hubble's original idea that in some way the morphological classification reflects an underlying physical sequence. In individual cases it may be difficult to estimate the morphological type accurately from visual inspection, but the results of expert classifiers agree quite well (Naim et al. 1995a). Although it is generally accepted that the morphological types form a sequence, it is not totally clear how sharp the boundaries are between successive types, nor whether the galaxy types indicate fundamental differences

^ Based on observations collected at the European Southern Observatory (La Silla, Chile).

$\star \star$ The full catalogue of galaxy types (Table 4) is only available in electronic form at the CDS via anonymous ftp to cdsarc.u-strasbg.fr (130.79.128.5) or via

http://cdsweb.u-strasbg.fr/cgi-bin/qcat?J/A+A/446/19 in several physical properties, or just a continuous change in a single physical quantity, such as e.g. bulge fraction (see van den Bergh 1997).

Studies of the color-magnitude relation (e.g. Bower et al. 1992; Ellis et al. 1997) and of mass-to-light ratios (e.g. Lucey et al. 1991; van Dokkum et al. 1996, 1998; Kelson et al. 2000) suggest that ellipticals and S0 galaxies form a single class of slowly evolving objects. From spectra, Jones et al. (2000) conclude that ellipticals and S0 galaxies have similar luminosityweighted ages and metal abundances. However, Kuntschner \& Davies (1998) obtained shorter luminosity-weighted ages for S0 galaxies in the Fornax cluster, and Smail et al. (2001) found shorter luminosity-weighted ages for the fainter S0 galaxies. At the same time, Dressler et al. (1997) and Fasano et al. (2000) found evidence that $\mathrm{S} 0$ galaxies have formed relatively recently (but see Andreon 1998 and Fabricant et al. 2000), and Poggianti et al. (1999), and Jones et al. (2000) even suggested that early spirals have transformed into S0 galaxies.

Poggianti et al. (1999) used the spectral catalogue of Dressler et al. (1999), of galaxies at intermediate redshifts $(z \sim 0.5)$, to study the connection between morphological and 
spectral transformations. They distinguish the following (spectral) galaxy types: starforming emission-line galaxies (ELG), post-starburst galaxies $(a+k / k+a)$ and passively evolving galaxies $(k)$. The fraction of post-starburst galaxies increases with redshift (Dressler \& Gunn 1983; Couch \& Sharples 1987). Poggianti et al. (1999) suggested that when individual galaxies entered clusters at intermediate redshifts they had a modest final starburst (see also Ellis et al. 2001; Ellingson et al. 2001) after which the galaxy evolved passively. These results agree with the finding that the fraction of gas-rich galaxies and the fraction of ELG decreases towards the cluster center (Biviano et al. 1997, Paper III; Solanes et al. 2001; Dale et al. 2001).

The transformation of a galaxy with starformation or even a modest starburst to a galaxy that is passively evolving will lead to changes in the structure of the galaxy. However, Poggianti et al. (1999) concluded that the morphological transformation, from early spiral to S0, cannot be driven purely by the star-formation history. Yet, the transformation of a significant number of early spirals into S0 galaxies, may be accompanied by (possibly subtle) spectral differences due to recent starformation. Therefore, it is of interest to study the average spectra of galaxies of different morphological types, using large samples of cluster galaxies.

We have determined the morphological types of close to 2300 galaxies in the ESO Nearby Abell Cluster Survey (ENACS, Katgert et al. 1996, 1998, Papers I and V of this series) from CCD images taken especially for this purpose. The majority of those galaxies (about 1450) had earlier been classified on the basis of their ENACS spectrum by de Theije \& Katgert (1999) (Paper VI). Following previous work (e.g. Zaritsky et al. 1995; Folkes et al. 1996; Lahav et al. 1996) we combined the morphological and spectral classifications into a single classification scheme. This is shown to be meaningful, and it increases the number of galaxies with "type"information, which is essential for the study of morphological segregation (Thomas \& Katgert 2006, Paper IX; Biviano et al. 2002, Paper XI). Finally, we study the average spectral properties of the galaxies in the various morphological classes.

This paper is organized as follows. In Sect. 2 we summarize the data. In Sect. 3 we investigate the consistency between our morphological types and those from the literature. In Sect. 4 we investigate the relation between galaxy morphology and the presence of emission lines in the spectrum. In Sect. 5 we compare the spectral and morphological types, and in Sect. 6 we discuss the combination of spectral and morphological types into one classification scheme. In Sect. 7 we compare the average spectra for the galaxies of the various morphological classes. Finally, we summarize and briefly discuss the main results in Sect. 8.

\section{The data}

Our analysis is based on data from the ENACS, which has yielded redshifts for 5634 galaxies in the directions of 107 nearby $(z \lesssim 0.1)$ Abell cluster candidates (see Papers I and V). The ENACS magnitudes are $R$-band, and the absolute magnitudes, $M_{R}$, were derived for $H_{0}=100 \mathrm{~km} \mathrm{~s}^{-1} \mathrm{Mpc}^{-1}$. Interlopers (non-members) were eliminated with the interloper removal procedure devised by den Hartog \& Katgert (1996) and applied to the ENACS dataset by Katgert et al. (2004, Paper XII). For 3798 galaxies in the ENACS sample, de Theije \& Katgert (Paper VI) used the ENACS optical spectrum to derive spectral galaxy types. The latter were "calibrated" with a subset of the galaxies for which Dressler (1980) had previously derived a morphological type. The spectral classification is based on the wavelength range from $372 \mathrm{~nm}$ to $501.4 \mathrm{~nm}$, which was observed at $\sim 0.6 \mathrm{~nm}$ resolution and sampled at $0.35 \mathrm{~nm}$. For a detailed description of the PCA/ANN-based spectral classification we refer to Paper VI.

In recent years, a long-term programme of CCD-imaging has been carried out with the 0.92-m Dutch telescope at La Silla. This has yielded $R$-band CCD-images for 2295 ENACS galaxies, from which we have derived morphological types. For $\sim 1450$ of those 2295 galaxies we can now compare the spectral classification from Paper VI with the new, CCD-based morphological type (this work). We must stress that the galaxies for which we have CCD-images are not a representative subset of the ENACS sample, because we selected relatively more early-type galaxies for our CCD-imaging programme.

The CCD that was used in the imaging had pixels of $0.44^{\prime \prime}$. Most observations were done in photometric conditions, and the seeing was between $1.2^{\prime \prime}$ and 2.0" (FWHM). To get reasonable signal-to-noise ratios for the galaxies, of which the apparent magnitudes in $R$ are brighter than 17, exposures of 3 min were made. Galaxies in the clusters A2361, A2401 and A2569 were observed with the 1.0-m JKT at La Palma. The latter observations were done with a similar resolution and seeing, but in non-photometric conditions.

\section{The morphological classification and its reliability}

We used the revised Hubble scheme (de Vaucouleurs 1959, 1963) to classify the galaxies by eye from their morphological appearance. The angular resolution of our data allowed us to assign galaxies to one of the following classes: E, E/S0, S0, $\mathrm{S} 0 / \mathrm{S}$, and $\mathrm{S}$. The E/SO and S0/S types are intermediate between $\mathrm{E}$ and S0, and S and S0 respectively. The spirals (S) are subdivided into early spirals (Se), late spirals ( $\mathrm{Sl}$ ) and generic spirals $(\mathrm{Sg})$. In our classification scheme the Se class comprises $\mathrm{Sa}, \mathrm{Sab}$ and $\mathrm{Sb}$ galaxies, and the S1 class Sbc, Sc, S/I and I (Irregular) galaxies. The $\mathrm{Sg}$ class consists of galaxies which we know are spirals, but for which no detailed classification was possible.

In our classification we used the criteria laid down by Dressler (1980). E galaxies have smooth (de Vaucouleurs) radial profiles without discontinuities in surface brightness. S0 galaxies have an additional non-spheroidal component (disk or lens). Spiral galaxies are (exponential) disk galaxies with spiral arms or ring patterns. The early spirals have brighter bulges and less open spiral arms than late spirals. The class of late spirals also contains irregular galaxies, i.e. amorphous objects with asymmetric isophotes.

Although the criteria used in the Hubble scheme are widely accepted, different authors are likely to have weighted these criteria in slightly different ways. In addition, the morphological 

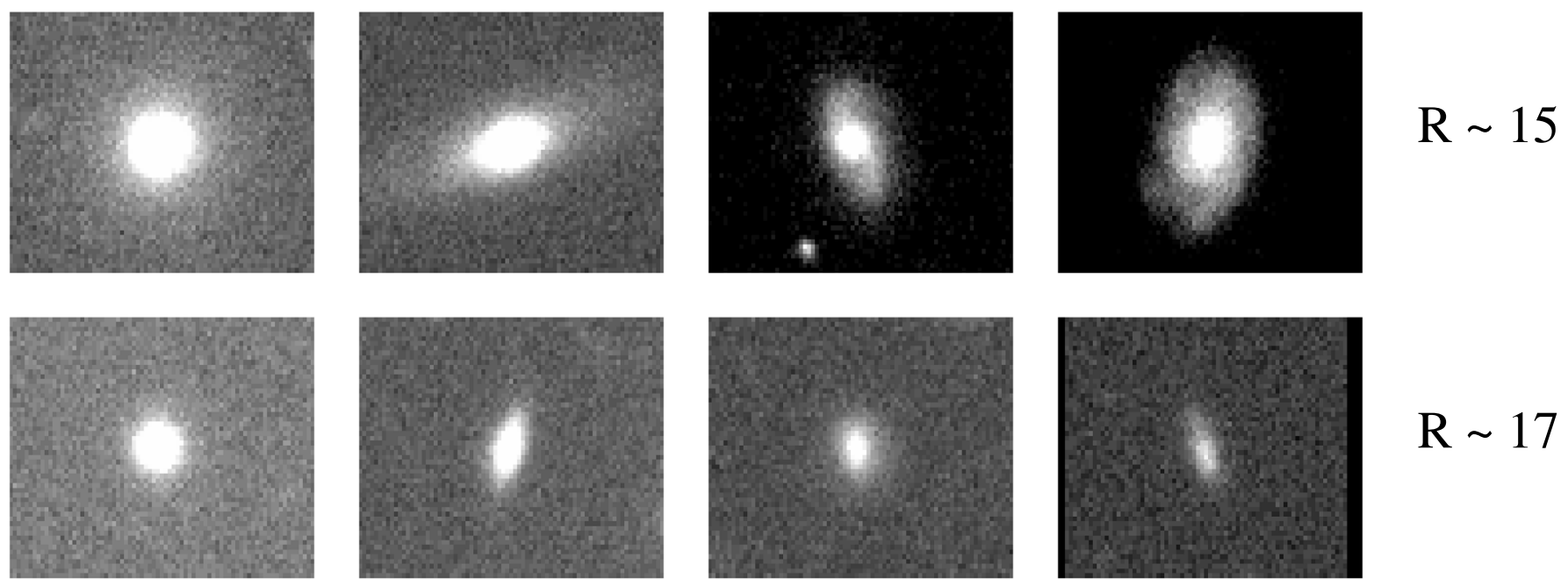

$\mathrm{R} \sim 17$
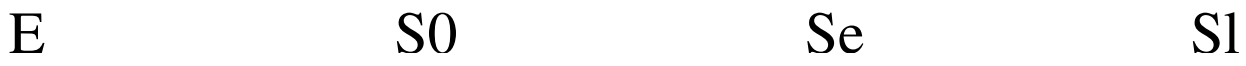

Fig. 1. Images of representative galaxies of all 4 morphological types, at two apparent magnitudes. Note that the grey scale contrasts are not identical in the 8 frames. As we explained in the text, for the fainter galaxies we sometimes used the brightness profile as additional evidence for the morphological classification.

Table 1. Number of galaxies per Hubble class; AD vs. TK.

\begin{tabular}{rc|rrrrrrr}
\hline \hline & & & \multicolumn{7}{|c}{ TK } \\
& & $\mathrm{E}$ & $\mathrm{E} / \mathrm{S} 0$ & $\mathrm{~S} 0$ & $\mathrm{~S} 0 / \mathrm{S}$ & $\mathrm{Sg}$ & Se & S1 \\
\hline & $\mathrm{E}$ & 71 & 12 & 11 & 1 & 0 & 0 & 0 \\
& $\mathrm{E} / \mathrm{S} 0$ & 13 & 4 & 6 & 0 & 0 & 0 & 0 \\
& $\mathrm{~S} 0$ & 18 & 19 & 106 & 25 & 6 & 2 & 1 \\
$\mathrm{AD}$ & $\mathrm{S} 0 / \mathrm{S}$ & 0 & 0 & 12 & 7 & 0 & 4 & 0 \\
& $\mathrm{Sg}$ & 0 & 0 & 2 & 9 & 16 & 19 & 6 \\
& $\mathrm{Se}$ & 0 & 0 & 1 & 9 & 3 & 47 & 2 \\
& $\mathrm{~S} 1$ & 0 & 0 & 0 & 0 & 1 & 3 & 12 \\
\hline
\end{tabular}

classification is not very quantitative, and it can depend on the inclination and the brightness of the galaxy. In particular, faceon S0 galaxies are difficult to distinguish from $\mathrm{E}$ galaxies. It is also difficult to distinguish edge-on S0 galaxies and early spirals since our images in general do not allow us to make statements about the presence of gas or dust. Likewise, early and late spirals are hard to distinguish if seen edge-on. In some cases, we used the 1-d brightness profile to help discrimate between $\mathrm{E}$ and S0 classification on the one hand and S0 and spiral classification on the other hand. In Fig. 1 we show images of representative galaxies of all 4 morphological types, at two apparent magnitudes. Note that the grey-scale contrasts are not identical in the 8 frames.

Naim et al. (1995a) made a comparative study of independent morphological classifications by various expert classifiers, in order to investigate the performance of their own, automated classification (Naim et al. 1995b). They numbered the successive broad Hubble classes E, S0, Sa+Sb, Sc+Sd and $\mathrm{Sm}+\mathrm{I}$, from 1 to 5 respectively, and determined the (cumulative) rate of agreement between different classifiers to within $n$ broad Hubble types. Among others, they compared Dressler's (1980) classification with both Buta's and Huchra's classifications. They concluded that the rates of agreement between classifications of different observers, and between those classifications and their automated classification, are very similar.

We compared our classification (TK) with that of Dressler (AD), for the 448 galaxies classified both by Dressler and by ourselves. In addition to these 448 galaxies, there are 138 galaxies with literature morphologies and with ENACS redshifts, but for which we do not have a CCD image. The numbers of galaxies in the AD-TK comparison are listed in Table 1. According to Table 1 the total rate of exact agreement between $\mathrm{AD}$ and TK (i.e., using all classes) is $59 \pm 4 \%$. Excluding the $\mathrm{E} / \mathrm{SO}$ - and S0/S-classes because these are not very meaningful for this comparison, we obtain $77 \pm 5 \%$. Finally, if we also exclude the indeterminate $\mathrm{Sg}$-class comparisons (i.e. $\mathrm{Sg}-\mathrm{Sg}$, $\mathrm{Sg}-\mathrm{Se}$ and $\mathrm{Sg}-\mathrm{Sl}$ ), we obtain $79 \pm 5 \%$ agreement. This latter number is the relevant one for all analyses, in which the S/S0, $\mathrm{E} / \mathrm{S} 0$ and $\mathrm{Sg}$ classes are not used.

In Fig. 2 we show the (cumulative) rate of agreement to within $n$ broad Hubble types, where we followed Naim et al. (1995a) and numbered the successive broad Hubble types as follows: $\mathrm{E}=1.0, \mathrm{E} / \mathrm{S} 0=1.5, \mathrm{~S} 0=2.0, \mathrm{~S} 0 / \mathrm{S}=2.5, \mathrm{Sa}+\mathrm{Sab}+$ $\mathrm{Sb}=3.0, \mathrm{Sg}=3.5, \mathrm{Sbc}+\mathrm{Sc}=4.0$ and $\mathrm{S} / \mathrm{I}+\mathrm{I}=5.0$. We show the rates of agreement between Dressler and Buta (AD-RB), between Dressler and Huchra (AD-JH), and between AD and TK, as a function of the difference between the two types. The 4 classification systems are seen to have very similar performance, with more than $80 \%$ of the comparisons showing a difference of not more than half a broad Hubble type.

For those 185 galaxies in common between AD and TK for which the two types are not identical (see Table 1), we adopted our morphological type, except in the following cases: (1) if the galaxy was classified as E/S0 by us and as E or S0 by AD, (2) if the galaxy was classified as S0/S by us and as S0 or Se by $\mathrm{AD}$, or (3) if the galaxy was classified as $\mathrm{Sg}$ by us and as $\mathrm{Se}$ or $\mathrm{Sl}$ by $\mathrm{AD}$. In addition, we adopted the morphological type given by Dressler (or by others) for those 138 galaxies in the 


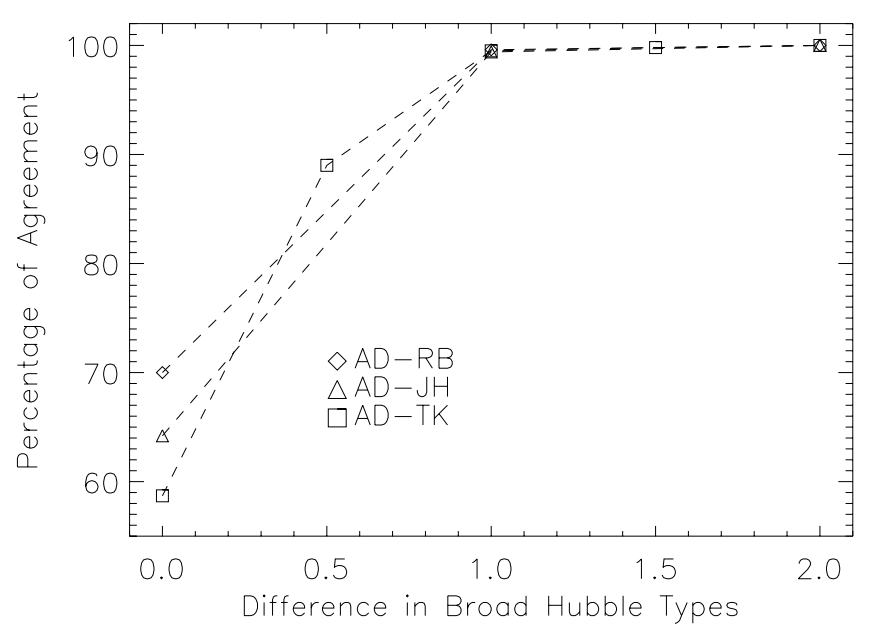

Fig. 2. The cumulative rate of agreement between the results of different classifiers, to within $n$ broad Hubble types. Clearly, the performance of the different classifications is very similar.

ENACS for which we do not have a CCD image and thus, no morphological type.

\section{The emission-line galaxies (ELG)}

Biviano et al. (1997, Paper III) discussed the presence of the [OII] $(372.7 \mathrm{~nm}), \mathrm{H} \beta(486.1 \mathrm{~nm})$ and the [OIII] doublet $(495.9+500.7 \mathrm{~nm})$ emission lines in the ENACS spectra. In the ENACS galaxies, these emission lines in general indicate the presence of warm gas, which is most likely connected to starformation. Galaxies with one or more of these emission lines are referred to as ELG.

In Table 2 we show the distribution of the ELG over morphological type for all galaxies in the ENACS sample. The fraction of ELG is very low for early-type galaxies $(4 \pm 1 \%$ for $\mathrm{E}$ galaxies and $5 \pm 1 \%$ for S0 galaxies) and rises from $27 \pm 4 \%$ for the early spirals to $59 \pm 4 \%$ for the late spirals. The latter implies that ELG and late spirals are very closely related. This is also evident in the analysis of the projected phase-space distributions, as described in Paper XI. ELG and late spirals without emission lines have very similar distributions, while they are significantly different from those of other galaxy types.

Because galaxies of different types have different projected distributions, one would expect the ELG and non-ELG to have different radial distributions. In Fig. 3 we show the relation between the fraction of ELG and the projected distance from the cluster center, for the early-type (E+S0) galaxies, the early spirals, the late spirals and all types together. We used the galaxies in the 67 clusters with at least 20 member galaxies and with $z<0.1$. Projected distances $R$ were scaled with $r_{200}$, the radius within which the average density of the cluster is 200 times as large as the critical density of the Universe, and which is a good approximation of the virial radius (e.g. Carlberg et al. 1997).

We used the Kolmogorov-Smirnov (or KS-) test to estimate the probability that the radial distributions of ELG and nonELG are drawn from the same parent distribution. According to this test the radial distributions of ELG and non-ELG are different $\left(P_{\mathrm{KS}}<0.01\right)$; i.e. the fraction of ELG decreases significantly towards the center. However, if we consider the Se or the SI galaxies separately, the fraction of ELG does not vary

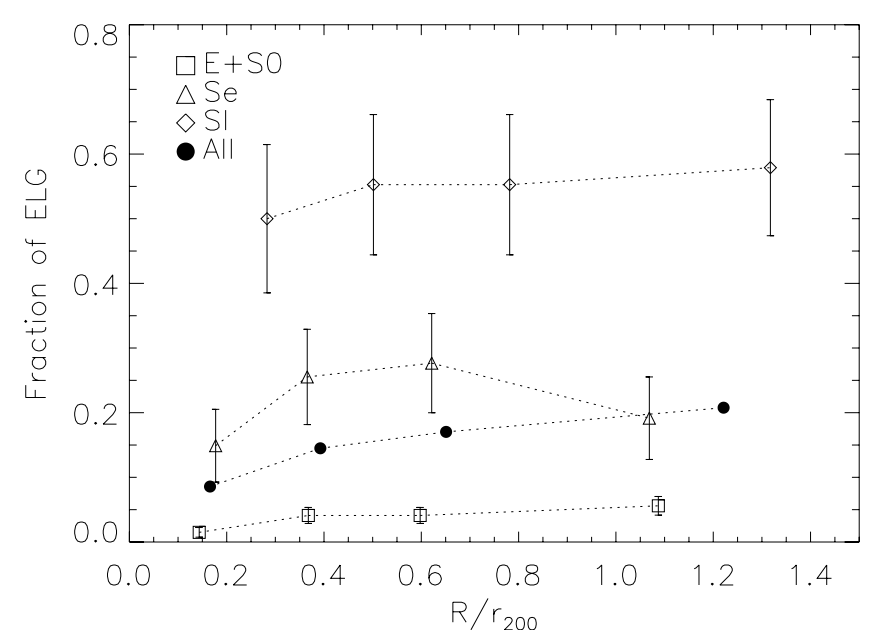

Fig. 3. The dependence of the fraction of ELG on the normalised projected cluster-centric distance $R / r_{200}$, for all galaxies in the 67 clusters with at least 20 member galaxies and with $z<0.1$ (filled circles). Also shown are the fractions for the individual galaxy classes.

Table 2. The distribution of ELG and non-ELG over morphological type.

\begin{tabular}{lrrr}
\hline $\begin{array}{l}\text { Morphological } \\
\text { type }\end{array}$ & ELG & non-ELG & ELG-fraction \\
\hline E & 16 & 344 & 0.04 \\
E/S0 & 9 & 139 & 0.06 \\
S0 & 40 & 763 & 0.05 \\
S0/S & 45 & 241 & 0.16 \\
Sa & 26 & 92 & 0.22 \\
Sab & 10 & 25 & 0.29 \\
Sb & 26 & 54 & 0.32 \\
Sg & 92 & 149 & 0.38 \\
Sbc & 12 & 13 & 0.48 \\
Sc & 33 & 42 & 0.44 \\
S/I & 34 & 22 & 0.61 \\
I & 54 & 14 & 0.79 \\
\hline All & 397 & 1898 & 0.17 \\
\hline
\end{tabular}

with $R$, so there is no significant difference between the distributions of ELG and non-ELG for these classes. Yet, there is a significant difference between the overall fractions of ELG for Se and Sl galaxies. This is in agreement with the result of Solanes et al. (2001), who concluded that gas is expelled more easily from early spirals than from late spirals. Apparently, the efficiency of the gas removal is more related to the type of spiral than to its position in the cluster.

\section{The spectral classification revisited}

De Theije \& Katgert (Paper VI) used a principal-component analysis (PCA) in combination with an artificial neural network (ANN) to classify most of the ENACS galaxies on the basis of their ENACS spectrum. They used the first 15 principal components in the ANN, of which the first describes the slope of the spectrum, and the second one the curvature; the following principal components describe several other features of the spectrum. The ANN was applied to a training set of 150 of the 270 galaxies for which morphological types (E, S0 or 


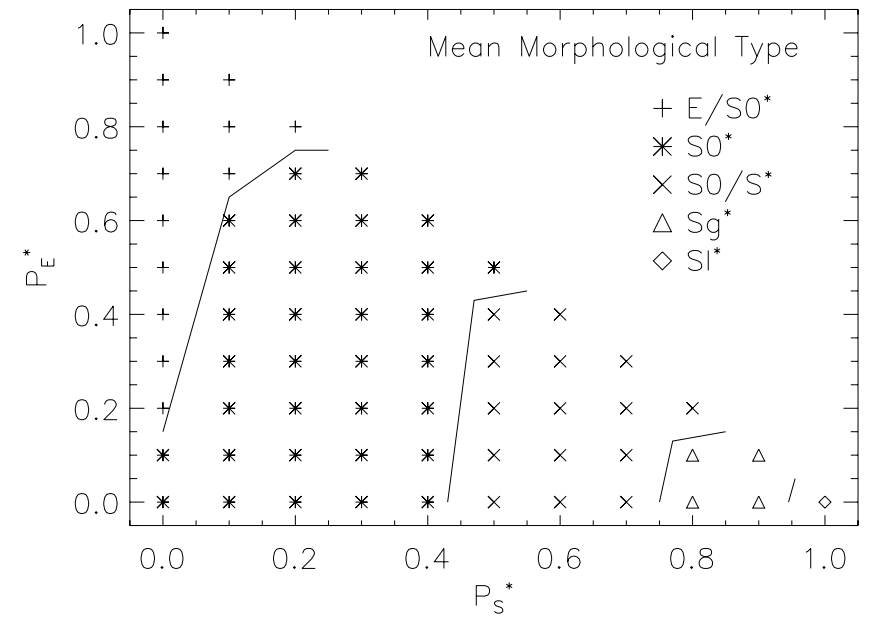

Fig. 4. For each of the 66 combinations of $P_{\mathrm{E}^{*}}$ and $P_{\mathrm{S}^{*}}$, as determined by de Theije \& Katgert from a PCA/ANN analysis of the ENACS spectra, we show the "mean" morphological type (see text).

S+I) were determined by Dressler (1980). To each combination of the 15 principal components, a spectral type $\left(\mathrm{E}^{*}, \mathrm{~S}^{*}\right.$ or $\left.(\mathrm{S}+\mathrm{I})^{*}\right)$ could be assigned, in such a way that the spectral and morphological types matched as closely as possible.

De Theije \& Katgert estimated the reliability of their spectral classification by repeating the ANN routine for 10 different, but - necessarily - correlated training sets. They thus obtained, for each galaxy, three probabilities; viz. $P_{\mathrm{E}^{*}}$, the number of times out of 10 that the galaxy was classified as an elliptical $\left(\mathrm{E}^{*}\right), P_{\mathrm{S}^{*}}$, the number of times out of 10 that the galaxy was classified as $(\mathrm{S}+\mathrm{I})^{*}$ and $P_{\mathrm{S}^{*}}\left(=1-P_{\mathrm{E}}^{*}-P_{\mathrm{S}}^{*}\right)$ the number of times out of 10 that the galaxy was classified as an $\mathrm{S}^{*}$.

Because the CCD-imaging program has greatly increased the number of galaxies with morphological types, we could check and recalibrate the PCA/ANN classification scheme of Paper VI with much better statistics. For each combination of $P_{\mathrm{E}^{*}}$ and $P_{\mathrm{S}^{*}}$ we derived the "mean" morphological type, which we will later use as the "spectral" type for that $\left(P_{\mathrm{E}^{*}}, P_{\mathrm{S}^{*}}\right)$ combination. However, in doing so we forced transitions from an earlier to a later type to occur only when $P_{\mathrm{S}^{*}}$ increases or $P_{\mathrm{E}^{*}}$ decreases. In Fig. 4 we show how the types, E/S0*, S0*, S0/S*, $\mathrm{Sg}^{*}$, and $\mathrm{Sl}^{*}$, vary with $P_{\mathrm{E}^{*}}$ and $P_{\mathrm{S}^{*}}$. It is clear from this Figure that $P_{\mathrm{S}^{*}}$ is the main factor driving the classification, while $P_{\mathrm{E}^{*}}$ appears to be only of secondary importance.

In Table 3 we show the relation between the morphological and spectral types, for the 1071 galaxies for which both types are available. Note that the table does not include galaxies with morphological types E/S0 or S0/S (about 220), while galaxies with a spectral type S0/S* (about 160) were not included either.

For 647 galaxies, the morphological and spectral types agree that the galaxy is of early type (i.e. E, E/S0 or S0), while for 240 galaxies both types indicate the galaxy to be of late type. So, the spectral classification can distinguish early-type and late-type galaxies with $83 \%$ reliability.

From Fig. 4 it is clear that the spectral classification has much more discriminating power for late-type galaxies than for early galaxy types. As a matter of fact, the "earliest" spectral type is not $\mathrm{E}^{*}$ but $\mathrm{E} / \mathrm{S}^{*}$. In the $\mathrm{E} / \mathrm{S}^{*}$ class, the morphological

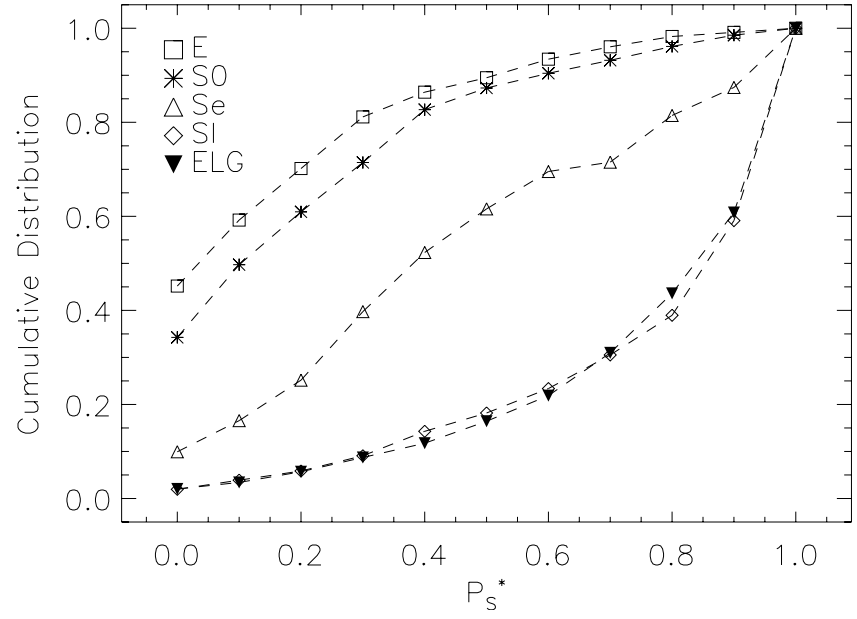

Fig. 5. The normalized cumulative $P_{\mathrm{S}}^{*}$-distributions of the 4 morphological galaxy classes and of the emission-line galaxies.

Table 3. The relation between morphological and spectral galaxy types.

\begin{tabular}{cc|rrrrr}
\hline \hline & & \multicolumn{5}{|c}{ Morphological type } \\
& & $\mathrm{E}$ & $\mathrm{S} 0$ & $\mathrm{Se}$ & $\mathrm{Sg}$ & $\mathrm{Sl}$ \\
\hline \multirow{3}{*}{ Spectral } & $\mathrm{E}^{\text {SO }} 0^{*}$ & 101 & 170 & 15 & 3 & 3 \\
type & $\mathrm{SO}^{*}$ & 96 & 280 & 64 & 35 & 19 \\
& $\mathrm{Sg}^{*}$ & 7 & 28 & 23 & 47 & 44 \\
& $\mathrm{Sl}^{*}$ & 2 & 8 & 19 & 44 & 63 \\
\hline
\end{tabular}

content is actually dominated by S0's (58\%) rather than by E's (35\%), but the fraction of E's in the E/S0* class is considerably larger than in the $\mathrm{S}^{*}$ spectral class (for which it is $19 \%$ ). The contamination by morphologically classified late-type galaxies in the $\mathrm{E} / \mathrm{SO}^{*}$ class is very small, viz. only $7 \pm 2 \%$. The $\mathrm{S}^{*}$ class is purer than the $\mathrm{E} / \mathrm{S}^{*}$ class, although contamination by ellipticals (19\%) and late-type galaxies (24\%) is far from negligible.

The late spectral types are divided in the $\mathrm{Sg}^{*}$ and $\mathrm{Sl}^{*}$ spectral classes, because our spectral classification scheme is not able to identify early spirals with any confidence. The $\mathrm{Sg}^{*}$ class contains more late spirals than early spirals, but the contamination of early-type galaxies is also non-negligible, at $23 \pm 2 \%$. The purest of all spectral classes is the $\mathrm{Sl}^{*}$ class. If we distribute the generic spirals over the early and late spiral classes, in the observed proportion of the morphological early and late spirals, we find that $72 \pm 5 \%$ of the galaxies in the $\mathrm{Sl}^{*}$ class have S1 morphologies.

Because the fraction of ELG varies with morphological type, we also compared the $P_{\mathrm{S}^{*}}$-distributions of all morphological classes, separately for non-ELG and ELG. The $P_{\mathrm{S}^{*-}}$ distributions of all 4 non-ELG morphological classes (E, S0, $\mathrm{Se}$ and $\mathrm{Sl}$ ) are different according to KS tests (i.e. have less than $1 \%$ probability to be drawn from the same distribution). The $P_{\mathrm{S}^{*}}$-distributions of the 3 ELG morphological classes (we did not consider the 13 ELG ellipticals) are not all different. The early and late spirals with emission lines do not have significantly different $P_{\mathrm{S}^{*}}$-distributions, but this negative result is probably due to limited statistics.

Although it is impossible to make a reliable distinction between ellipticals and S0 galaxies on the basis of the spectrum 
alone, ellipticals have somewhat earlier spectral types than S0 galaxies. This is evident in Fig. 5, which shows the $P_{\mathrm{S}^{*}}$ distributions of the various morphological classes. Although the $P_{\mathrm{S}^{*}}$-distributions of the $\mathrm{E}$ and $\mathrm{S} 0$ galaxies are very similar, a KS-test shows them to have less than $1 \%$ probability to be drawn from the same parent distribution. From Fig. 5 it is also clear that the late spirals and the ELG are spectroscopically indistinguishable, while the spectra of both are very different from those of early spirals and E and S0 galaxies. The great spectral similarity of late spirals and ELG has led us to classify all generic spectroscopic spirals (i.e. $\mathrm{Sg}^{*}$ ) with emission lines as $\mathrm{Sl}^{*}$. By the same token, ELG for which no PCA/ANN spectral type could be obtained were classified as $\mathrm{Sl}^{*}$.

\section{Combining morphological and spectral types}

For 2433 galaxies we have a morphological type: 1847 of those had the type determined only from the present CCD imaging; another 138 only have a type from the literature, while for the remaining 448 there are morphological types from the literature as well as from the present CCD imaging. For 185 of the latter, the two morphological types were not identical (although often consistent), and for those we adopted our own type, except in the few cases described at the end of Sect. 3.

Revised spectral types (see Sect. 5) were available for 3798 galaxies. In addition there are 421 galaxies for which no spectral type was available (primarily because the de-redshifted wavelength interval of the ENACS spectrum did not have sufficient overlap with the standard range adopted in the PCA/ANN analysis - see Paper VI), but for which the spectrum was inspected and found to contain one or more emission lines (see Paper III). This latter (ELG) information is less specific about galaxy type than the PCA/ANN spectral type. Yet, it was sometimes used to assign the spectral type $\mathrm{Sl}^{*}$, viz. if there was no PCA/ANN spectral type, or if the spectral type was $\mathrm{Sg}^{*}$.

We have combined the morphological and (recalibrated) spectral types into a single set of 4879 galaxy types in the following way. For about half of the galaxies, we only have a spectral type and that was then adopted as the galaxy type. For the other half, we had a morphological type and sometimes a spectral type. For those, the morphological type was adopted, except in those cases where the spectral type could help resolve an ambiguous or non-specific morphological type, like E/S0, $\mathrm{S} 0 / \mathrm{S}$ or $\mathrm{Sg}$.

If the morphological type was E/SO and the spectral type was $\mathrm{S}^{*}$ the galaxy was classified as $\mathrm{S} 0$. If the morphological type was $\mathrm{S} 0 / \mathrm{S}$ and the spectral type was $\mathrm{E} / \mathrm{S}^{*}$ or $\mathrm{S}^{*}$ the galaxy was also classified as S0. If the morphological type was S0/S and the spectral type was $\mathrm{Sg}^{*}$, while the galaxy had $P_{\mathrm{S}^{*}}<0.9$, it was classified as Se. Similarly, we tried to make the morphological Sg type more specific, as follows. If the spectral type was $\mathrm{E} / \mathrm{S}^{*}$, $\mathrm{S}^{*}$ or $\mathrm{S} 0 / \mathrm{S}^{*}$, the adopted galaxy type was Se because $75 \%$ of the spirals in these spectral classes have Se morphologies. Likewise, if the spectral type was $\mathrm{Sl}^{*}$ or $\mathrm{Sg}^{*}$ with $P_{\mathrm{S}}^{*} \geq 0.9$, the adopted galaxy type was $\mathrm{Sl}$, because $77 \%$ of the spirals in this spectral class have Sl morphologies.

This procedure yields a consistent classification for 4879 ENACS galaxies. For 2102 (i.e. 43\%) of those,

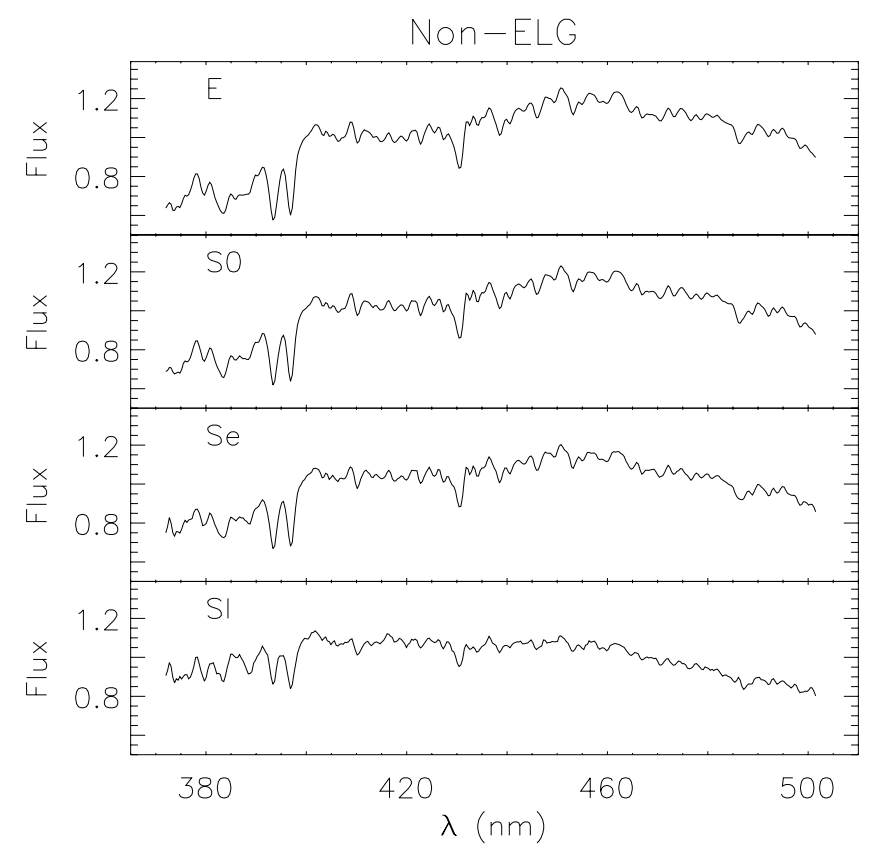

Fig. 6. The mean spectra for ellipticals (E), S0 galaxies, early spirals (Se) and late spirals (Sl), without emission lines (non-ELG). The mean spectrum was determined by averaging individual spectra in which the mean flux was normalised to one (arbitrary units). The individual galaxy spectra were not spectro-photometrically calibrated, but have been corrected for the spectral response of spectrograph and detector.

the morphological type was adopted; for 2446 (i.e. 50\%) the spectral type was adopted, while for the remaining 331 (i.e. $7 \%$ ) of the galaxies, the morphological and spectral types were combined, as described above.

The results for the 4879 galaxies are available at the CDS in Strasbourg. The final galaxy types that we used are: E, E/S0 (could be either), S0, S0/S (could be either), Se (could be Sa, $\mathrm{Sab}, \mathrm{Sb}$, and sometimes the morphological type is indeed more specific), Sl (could be Sbc, Sc, S/I and I, and sometimes the morphological type is indeed more specific) and $\mathrm{Sg}$ (generic spiral without information on subtype). In Table 4 we show, as an example, the results for the 44 galaxies in cluster Abell 13. For each galaxy we give the number (as in the original ENACS catalogue - see Katgert et al. 1998, Paper V), the morphological and spectral galaxy type, and the ELG character (when applicable) together with the final galaxy type adopted in subsequent papers, obtained as described above.

\section{The average spectral properties of the various galaxy classes}

The PCA appears to be a good tool to distinguish between spectra, but the physical meaning of most of the principal components, and especially of the higher-order ones, is not intuitively clear. We have tried to get a better understanding of the spectral differences between the various morphological types by studying the average spectra of the different galaxy classes. 
Table 4. The catalogue of galaxy types (extract; the full catalogue is available electronically at the CDS).

\begin{tabular}{|c|c|c|c|c|c|c|c|c|c|c|c|}
\hline \multirow{2}{*}{$\begin{array}{c}\text { Cluster } \\
\text { name }\end{array}$} & \multirow{2}{*}{$\begin{array}{c}\text { galaxy } \\
\text { nr. }\end{array}$} & \multicolumn{3}{|c|}{ galaxy type } & \multirow[t]{2}{*}{ 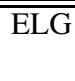 } & \multirow{2}{*}{$\begin{array}{c}\text { Cluster } \\
\text { name }\end{array}$} & \multirow{2}{*}{$\begin{array}{c}\text { galaxy } \\
\text { nr. }\end{array}$} & \multicolumn{3}{|c|}{ galaxy type } & \multirow[t]{2}{*}{$\overline{\overline{E L G}}$} \\
\hline & & morph. & spectral & adopted & & & & morph. & spectral & adopted & \\
\hline A0013 & 1 & S0/S & S0 & S0 & & A0013 & 23 & & S1 & S1 & $*$ \\
\hline A0013 & 2 & & So & So & & A0013 & 24 & S0 & E/S0 & S0 & \\
\hline A0013 & 3 & & $\mathrm{Sg}$ & $\mathrm{Sg}$ & & A0013 & 25 & S0 & S0 & S0 & \\
\hline A0013 & 4 & So & So & So & & A0013 & 26 & & S0/S & S0/S & $*$ \\
\hline A0013 & 5 & & Sl & $\mathrm{Sl}$ & $*$ & A0013 & 27 & So & $\mathrm{E} / \mathrm{S} 0$ & So & \\
\hline A0013 & 6 & So & So & So & & A0013 & 28 & & $\mathrm{Sg}$ & $\mathrm{Sg}$ & \\
\hline A0013 & 7 & & S0 & S0 & & A0013 & 29 & S0 & E/S0 & S0 & \\
\hline A0013 & 8 & $\mathrm{E}$ & $\mathrm{E} / \mathrm{S} 0$ & E & & A0013 & 30 & S0 & E/S0 & S0 & \\
\hline A0013 & 9 & & S0/S & S0/S & & A0013 & 31 & & Sl & Sl & \\
\hline A0013 & 10 & & Sl & Sl & $*$ & A0013 & 32 & So & So & So & \\
\hline A0013 & 11 & $\mathrm{Sa}$ & S0 & $\mathrm{Se}$ & & A0013 & 33 & So & So & So & \\
\hline A0013 & 12 & $\mathrm{E}$ & $\mathrm{E} / \mathrm{S} 0$ & E & & A0013 & 34 & $\mathrm{Sa}$ & Sl & $\mathrm{Se}$ & $*$ \\
\hline A0013 & 13 & So & $\mathrm{E} / \mathrm{S} 0$ & So & & A0013 & 35 & E/S0 & $\mathrm{E} / \mathrm{S} 0$ & $\mathrm{E} / \mathrm{S} 0$ & \\
\hline A0013 & 14 & & S0 & So & & A0013 & 36 & S0 & So & So & \\
\hline A0013 & 15 & $\mathrm{Sa}$ & So & $\mathrm{Se}$ & & A0013 & 37 & & $\mathrm{Sg}$ & $\mathrm{Sg}$ & \\
\hline A0013 & 16 & So & S0 & S0 & & A0013 & 38 & $\mathrm{E}$ & So & $\mathrm{E}$ & \\
\hline A0013 & 17 & So & So & So & & A0013 & 39 & $\mathrm{~S} 0 / \mathrm{S}$ & E/S0 & So & \\
\hline A0013 & 18 & & $\mathrm{~S} 0 / \mathrm{S}$ & S0/S & & A0013 & 40 & & $\mathrm{Sg}$ & $\mathrm{Sg}$ & \\
\hline A0013 & 19 & $\mathrm{E}$ & $\mathrm{E} / \mathrm{S} 0$ & $\mathrm{E}$ & & A0013 & 41 & $\mathrm{Sg}$ & Sl & Sl & $*$ \\
\hline A0013 & 20 & $\mathrm{E} / \mathrm{S} 0$ & $\mathrm{E} / \mathrm{S} 0$ & $\mathrm{E} / \mathrm{S} 0$ & & A0013 & 42 & & S1 & S1 & $*$ \\
\hline A0013 & 21 & & $\mathrm{~S} 0 / \mathrm{S}$ & $\mathrm{S} 0 / \mathrm{S}$ & & A0013 & 43 & & S0/S & S0/S & $*$ \\
\hline A0013 & 22 & & So & S0 & & A0013 & 44 & & S0 & S0 & \\
\hline
\end{tabular}

\subsection{The average spectra of the non-ELG}

In Fig. 6 we show the mean spectra for the 4 non-ELG classes. The mean spectrum for each class was determined by averaging individual spectra in which the mean flux was normalized to one (arbitrary units). The spectra were not calibrated spectrophotometrically, but the spectral response of the grating and the CCD was corrected as described in Paper VI. Although one could argue that the average of the spectra of many galaxies does not necessarily have a clear physical meaning, Fig. 6 illustrates the well-known global spectral differences between late spirals and early-type galaxies, both as regards spectral slope and strength of the absorption lines.

Because color has been shown to depend on luminosity (e.g. Bower et al. 1992), while luminosity-weighted ages were found to increase from faint to bright SO galaxies (see e.g. Smail et al. 2001), we also compared the average spectra of "faint" and "bright" non-ELG galaxies. For this comparison, we estimated the magnitude limit between "faint" and "bright" galaxies in the following way. For various limits in absolute magnitude we compared the $P_{\mathrm{S}^{*}}$-distributions of non-ELG galaxies fainter and brighter than this limit. For limits between $M_{R}=-19.5$ and $M_{R}=-20.0\left(H_{0}=100 \mathrm{~km} \mathrm{~s}^{-1} \mathrm{Mpc}^{-1}\right)$ the $P_{\mathrm{S}^{*}}$-distributions of faint and bright $\mathrm{S} 0$ galaxies are different according to KS-tests. Because the number of "faint" galaxies is relatively small, we chose $M_{R}=-20$ as the magnitude limit. In Table 5 we give the number of "bright" and "faint" galaxies for each type.

In Fig. 7 we show the differences between the mean spectra of faint $\left(M_{R}>-20\right)$ and bright $\left(M_{R} \leq-20\right)$ galaxies for the 4 non-ELG classes. A positive slope in this figure corresponds to a bluer continuum of the first (fainter) galaxy class.
Table 5. The number of non-ELG galaxies for which we determined morphological type, "colors" and "linestrengths".

\begin{tabular}{c|rrrr}
\hline \hline & \multicolumn{4}{|c}{ Morphological Type } \\
& $\mathrm{E}$ & S0 & Se & Sl \\
\hline Bright $\left(M_{R} \leq-20\right)$ & 203 & 326 & 91 & 41 \\
Faint $\left(M_{R}>-20\right)$ & 27 & 207 & 25 & 20 \\
\hline Total & 230 & 533 & 116 & 61 \\
\hline
\end{tabular}

Therefore, the bright galaxies in general have a redder continuum than the faint ones. This conclusion, which is wellestablished for the early-type galaxies (see, e.g., Bower et al. 1992), also appears to be true for the spirals.

We quantified these differences by determining pseudocolors " $b-v$ " and " $v-g$ ", where the " $b$ "-, " $v$ "- and " $g$ "-band magnitudes were defined as the median magnitudes in the spectral ranges 372 to $389.5 \mathrm{~nm}, 405$ to $422.5 \mathrm{~nm}$, and 483.9 to $501.4 \mathrm{~nm}$ respectively. It appears that " $b-v$ " and " $v-g$ " are rather insensitive to the presence of emission lines, but " $b-v$ " is sensitive to the strength of the $400 \mathrm{~nm}$ break. For each galaxy class we determined the mean colors by averaging the estimates of individual galaxies. The uncertainty in the mean colors was determined by dividing the dispersion of the individual estimates within a galaxy class by the square root of the number of galaxies (see Table 5). The "uncertainty" therefore derives primarily from the intrinsic spread within a class.

The results are shown in Fig. 8, which shows the mean colors $\langle " b-v "\rangle$ and $\langle " v-g$ " $\rangle$ for the bright and faint subsets of each of the 4 galaxy classes. The most obvious effect in this figure is that the colours get bluer towards later Hubble type. For the bright galaxies, the effect is significant at $3 \sigma$ or more for all comparisons between consecutive Hubble classes, and 


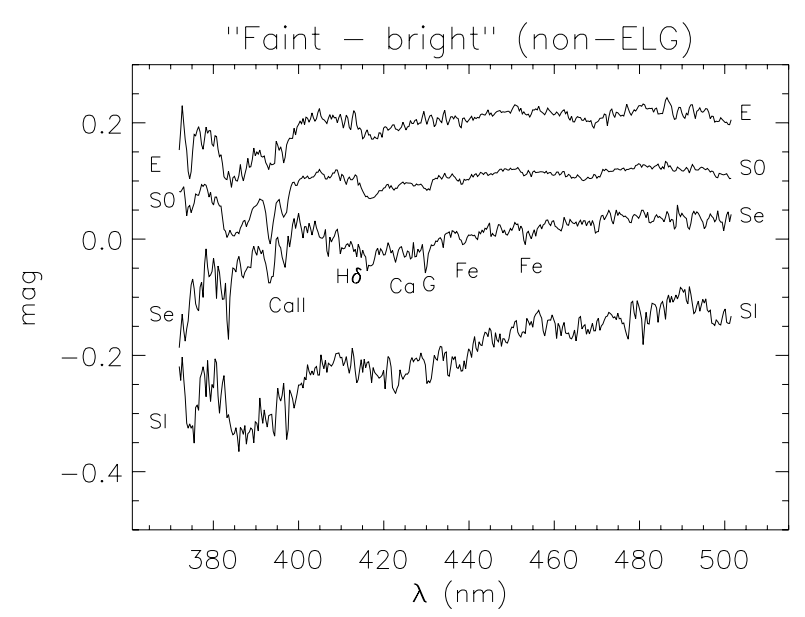

Fig. 7. The difference (in magnitudes) between the spectra of the faint $\left(M_{R}>-20\right)$ and bright $\left(M_{R} \leq-20\right)$ non-ELG galaxies, for different morphologies. The difference spectra have zero mean flux, but were shifted so that they could be shown in one figure.

in both colors. For the faint galaxies, the same trend is visible, albeit that the statistical weight (and therefore, the significance) is considerably less. The great similarity between the variation of $\langle " b-v "\rangle$ and $\langle " v-g "\rangle$ with galaxy type, indicates that the two colors are strongly correlated. This confirms that the depth of the $400 \mathrm{~nm}$ break is strongly correlated with the continuum slope.

At the same time, there is a tendency for the bright galaxies in each class to be redder than the faint ones. The effect is significant at $3 \sigma$ or more for the S0 galaxies in $\langle “ b-v "\rangle$, and for the late spirals in both colors. The latter result may be somewhat unexpected, and in interpreting it one must remember that the colors (as derived from the ENACS spectra) are valid for a central region of 3-6 kpc diameter, and thus do not refer to the entire galaxy. With some exaggeration, one may therefore conclude that the color-magnitude relation that is well-established for early-type galaxies even extends to the bulges and the central parts of the disks of late-type galaxies. Without further data it is impossible to correct for the disk contribution.

We have also estimated the strengths of the most prominent absorption lines, viz.: CaII $393.4+396.8 \mathrm{~nm}, \mathrm{H} \delta 410.2 \mathrm{~nm}$, CaI $422.7 \mathrm{~nm}$, G-band $430.4 \mathrm{~nm}$, FeI $438.3 \mathrm{~nm}$ and FeI $453.1 \mathrm{~nm}$, and of the following emission lines: OII $372.7 \mathrm{~nm}$, $\mathrm{H} \beta 486.1 \mathrm{~nm}$ and OIII 495.9+500.7 nm. For each line we determined the total flux, $F_{1}$, in the central 5 pixels of the line ( $\sim 1.8 \mathrm{~nm}$ or $\sim 3 F W H M$ of the instrumental profile), and we determined the continuum flux, $F_{\mathrm{c}}$, in 6 pixels outside these central 5 pixels ( 3 pixels on each side). We defined the linestrength as $\Delta m=-2.5 \log F_{1} / F_{\mathrm{c}}$. Because the $\mathrm{CaI} 422.7 \mathrm{~nm}$, FeI $438.3 \mathrm{~nm}$ and FeI $453.1 \mathrm{~nm}$ lines are relatively weak, we improved the signal-to-noise ratio by averaging their strengths into a single value for each galaxy. Note that our $\Delta m$-values cannot be compared directly to equivalent widths (EW). This is because we could not fit the line profile with a model (e.g. a Gaussian) because of the limited resolution of our data.

In the upper 3 panels of Fig. 9 we show the average strengths of the absorption lines (as defined above) for the brighter and fainter subsets of the different galaxy classes. The

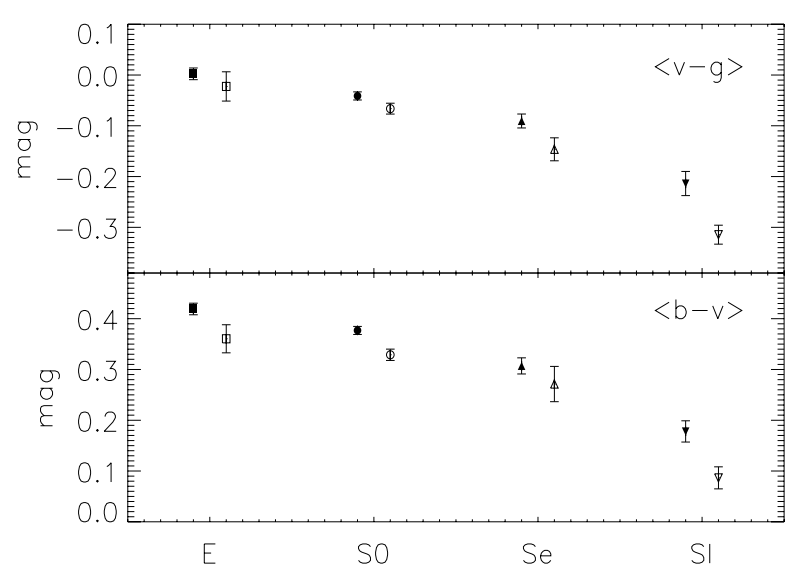

Fig. 8. The mean colors $\langle " v-g$ " (top) and $\langle " b-v "\rangle$ (bottom) of the various morphological classes. Note that the colors are instrumental, because the spectra were not spectro-photometrically calibrated. For each of the 4 galaxy classes we show the colors for bright $\left(M_{R} \leq\right.$ $-20)$ galaxies (filled symbols) and faint $\left(M_{R}>-20\right)$ galaxies (open symbols) separately.

CaII, G-band and FeI+CaI lines are indicators of metallicity. The uncertainty in the mean linestrengths was determined by dividing the dispersion of the individual estimates within a galaxy class by the square root of the number of galaxies (see Table 5). Again, the "uncertainty" therefore mostly measures the intrinsic spread within a class.

As with the colors, there is a trend in Fig. 9 for the metalline strengths to decrease towards later Hubble type. However, the effect is significant at $3 \sigma$ or more for the comparison between early and late spirals only, and in that case it is significant for all 3 metal lines and for bright as well as faint subsets. There is also a tendency (certainly among the S0 galaxies and the early and late spirals) for the metal-lines to be stronger for the bright galaxies than for the faint ones. The difference is significant at $3 \sigma$ or more for CaII in S0 galaxies only, and for G-band and $\mathrm{FeI}+\mathrm{CaI}$ for the late spirals only.

In Fig. 10 we have combined the information on color and strength of the three metal lines. Because the $\langle " v-g$ " $\rangle$ and $\langle " b-v "\rangle$ colors are very strongly correlated, we only show the correlation with $\langle$ " $v-g$ " $\rangle$ color. For this figure we have chosen to combine the 3 metal-line strengths. Because the measured strengths of the G-band and FeI+CaI lines are small, we approximately brought them on the scale of the CaII linestrength by multiplying them by constant factors (viz. 1.68 and 4.57 respectively). Subsequently, we averaged the 3 line-strengths to produce the value displayed in Fig. 10.

This figure summarizes in a compact way the properties of the average spectra of the non-ELG that we discussed so far. There is a strong and significant correlation between $\langle " v-g$ " $\rangle$ color and metallicity, with a very systematic decrease of metallicity and blueing of the color towards late Hubble type. In addition, the faint galaxies in each class are generally bluer and less metal-rich than the bright galaxies. Although both effects are not always significant at the $3 \sigma$-level in individual comparisons, the general trends are very strong. One interesting result, which may be significant or fortuitous, is that colors and metallicities of faint S0 galaxies and bright early spirals appear to be very similar. 


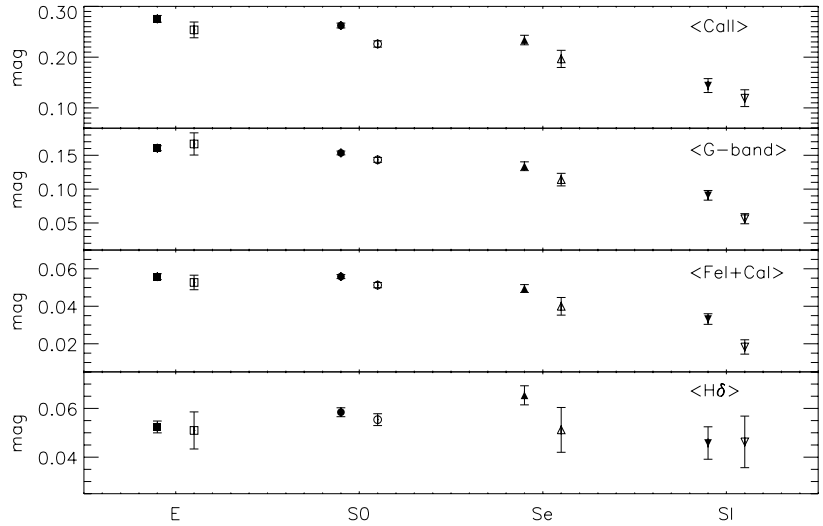

Fig. 9. The strengths of the absorption lines. Filled and open symbols refer to the bright and faint subsets of the 4 galaxy classes. The upper 3 panels show the strengths of the 3 metal line(-complexe)s, and the lower panel the strength of the $\mathrm{H} \delta$ line. For the definition of the linestrength $\Delta m$, see the text; absorption-line strength increases upwards.

In the bottom panel of Fig. 9 we show the strength of the $\mathrm{H} \delta$ absorption line for the different galaxy types, and luminosity classes. The $\mathrm{H} \delta$ absorption line is often used as an indicator for recent star-formation (e.g. Dressler et al. 1999). There are no differences of $3 \sigma$ or more between the different morphological types, neither for the bright nor faint subclasses. Yet, there may be a tendency for the bright $\mathrm{S} 0$ and, in particular, the bright early spirals to have stronger $\mathrm{H} \delta$ absorption than do the ellipticals and the late spirals. The fact that the bright early spirals have the strongest $\mathrm{H} \delta$ absorption may be due to enhanced recent starformation that some bright early spirals have undergone (e.g. Poggianti et al. 1999).

One may wonder if our result, that the bright early spirals in local clusters may have a slightly higher $\mathrm{H} \delta$ absorption-line strength is in conflict with the result obtained by Poggianti et al. (2004), who found that the fraction of luminuous $(a+k / k+a)$ galaxies is much smaller in the Coma cluster than it is in the $z \sim 0.5$ clusters studied by Dressler et al. (1999). However, the identification between their $(a+k / k+a)$ galaxies (spectroscopically defined) and our early spirals (morphologically defined) is quite doubtful. For one thing, the average $\mathrm{H} \delta$ equivalent width of our (bright) early spirals is unlikely to be as high as that of the high-redshift $(a+k / k+a)$ galaxies. The only thing one might conclude is that the fraction of $(a+k / k+a)$ galaxies is slightly higher among the bright early spirals than it is on average.

\subsection{The average spectra of the ELG}

In Fig. 11, we show the differences between the average spectra of non-ELG and ELG for each of the 4 galaxy classes. The non-ELG have significantly redder continuum colors and stronger absorption lines than the ELG ( $>3 \sigma$-result), and this is true for all galaxy types. To within the "uncertainties" (which are mostly due to the spread within a morphological class) the differences between the mean colors and mean absorption-line strengths of non-ELG and ELG are indistinguishable for E, S0, Se and Sl galaxies.

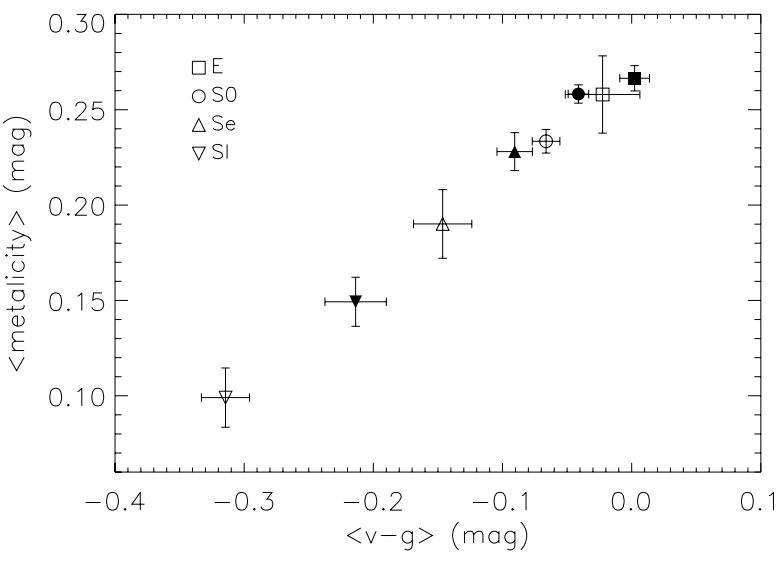

Fig. 10. The relation between color and metallicity. Because the $\langle$ " $v-$ $g "\rangle$ and $\langle " b-v "\rangle$ colors are very strongly correlated, we only show the correlation with $\langle " v-g$ " $\rangle$ color. The metallicity was derived by combining the three measured line-strengths as described in the text. Note that the combined metallicity is given on the CaII-scale.

This independence of the mean (non-ELG - ELG) difference spectrum of morphological type is quite remarkable, even though in second order there are rather subtle differences in linestrength, while it must also be kept in mind that the spread within each morphological class is considerable (with a median spread of $\Delta m \approx 0.08$ for the non-ELG and $\Delta m \approx 0.10$ for the ELG). Taken at face value this independence implies that the process that is responsible for the emission lines appears to produce a blueing of the continuum of the galaxy, by an average amount that is very similar for all galaxy types. An explanation for this could be that the primary source for the excitation of the lines is most likely photo-ionization.

If one combines this result with the conclusions about the type-dependence of the colors of non-ELG (as summarized in Fig. 8) it seems likely that the latter apply also to the ELG of the different morphological classes. However, it should be remembered that galaxies with weaker emission lines may not have been recognized as ELG, and those ELG that went unrecognized will have been included in the non-ELG class. This means that, if such ELG (i.e. with emission lines below our detection limit) do exist and if they also have a bluer continuum than the true non-ELG, the difference between ELG and non-ELG could be larger than shown in Fig. 11.

In view of the considerably smaller statistics of (in particular, the early-type) ELG, we refrain from a detailed analysis of the strengths of absorption and emission lines in the spectra of the various morphological classes among the ELG. We find only one significant effect, viz. that the $\mathrm{H} \beta$ (486.1 nm) emission line is $0.09 \pm 0.03$ mag brighter in the average spectrum of the spiral ELG than it is in that of the SO ELG.

\section{Discussion and conclusions}

We used CCD-images to determine the morphological types of 2295 galaxies in the ENACS sample. From a comparison with morphological types derived by Dressler (1980) for a subset of these galaxies, we conclude that the reliability of our morphological classification is very comparable to that of Dressler 


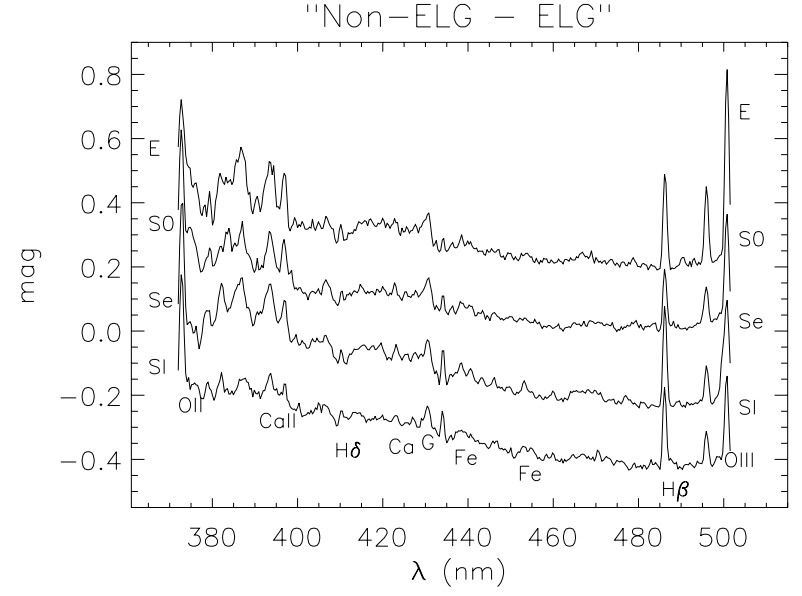

Fig. 11. The ratio (in magnitudes) between the mean non-ELG and ELG spectra for different morphological classes. The residual spectra all have identical (i.e. zero) mean flux, but they were offset to be shown in one figure.

and, by implication, to that of other expert classsifiers. With these new morphologies we could show that the spectral classification of Paper VI (based on the ENACS spectra), allows the broad distinction between early- and late-type galaxies to be made with a reliability of $83 \pm 1 \%$. However, within the earlytype class, the spectral classification can barely distinguish ellipticals from S0 galaxies. On the contrary, late spirals can be classified from the spectrum alone with $72 \pm 5 \%$ reliability.

We compared the average spectral properties of galaxies without emission lines (non-ELG) with different morphologies and luminosities, and we reached the following conclusions.

The continuum colors, and the strength of the metal lines are strongly and significantly correlated, with a very systematic decrease of metallicity and a blueing of the color towards late Hubble type. In addition, the faint galaxies in each class are generally bluer and less metal-rich than the bright galaxies. For the colors the latter is a confirmation of the well-established color-magnitude relation found previously for early-type galaxies (see e.g. Bower et al. 1992). However, our data show that this relation also extends to the spirals. It is perhaps noteworthy that, within this very strong global trend, the colors and metallicities of faint S0 galaxies and bright early spirals are very similar.

Peletier et al. (1999) found that the bulges of nearby early spirals have colors at their half-light radii that are very similar to those of the ellipticals in Coma, which suggests that bulges of ellipticals and early spirals are similar. However, early spirals have bluer colors than ellipticals, which implies that the disk must contribute significantly. Our observations show that galaxies of later Hubble types and galaxies of lower luminosities indeed have bluer continua and weaker metal lines. At the same time the average bulge fraction decreases towards later types (see e.g. Kent 1985; Simien \& de Vaucouleurs 1986; Schechter \& Dressler 1987) and towards lower luminosities (e.g. Gavazzi et al. 2000). The spectral properties of galaxies might therefore be mainly correlated with the bulge fraction.

The bright early spirals on average have somewhat stronger $\mathrm{H} \delta$ absorption than the other galaxies, although in our data the effect is at the $\sim 2 \sigma$-level only. The $\mathrm{H} \delta$ absorption-line is an indicator for recent starformation (e.g. Dressler et al. 1999). Observations of galaxies in clusters at $z \sim 0.5$ have shown that in many galaxies star formation was quenched after a final starburst (e.g. Dressler \& Gunn 1983; Couch \& Sharples 1987), which probably occurred when galaxies entered the cluster (Poggianti et al. 1999; Ellingson et al. 2001). Poggianti et al. concluded that the transformation from spirals to S0 galaxies occurred after the starburst phase and on longer time scales, because the observed post-starburst galaxies were mainly spirals instead of S0 galaxies. It is thus possible that the transformation of early spirals into S0 galaxies is presently still going on, albeit probably at a fairly low level.

The analysis of the properties of the galaxies with emission lines (ELG) gives the following results.

The continuum spectrum of the ELG is bluer than that of the non-ELG, and the blueing is, to first order, identical for galaxies of all morphological types. This could imply that the primary source for the line-excitation is photo-ionization

The fraction of ELG depends strongly on galaxy type: from $4 \pm 1 \%$ for ellipticals to $59 \pm 4 \%$ for late spirals (including irregular galaxies). This is consistent with the result obtained in Paper III, which was based on the subset of ENACS galaxies with Dressler morphological types (about a factor of 4 smaller than the present sample). It is also consistent with the finding by Solanes et al. (2001) that the fraction of gas-rich galaxies increases strongly towards late Hubble types. According to Solanes et al. this indicates that gas is removed more easily from early spirals than from late spirals.

On the contrary, for each morphological type, the fraction of ELG varies relatively little with projected distance from the cluster center. This is consistent with the conclusion of Dale et al. (2001), who found that the fraction of gas-rich galaxies decreases only slightly towards the dense cluster center. Apparently, the efficiency of the gas removal is more related to the type of galaxy than to its position in the cluster. However, one cannot conclude from this result that the efficiency of the process by which the gas is expelled is independent of position within the cluster. This is because the removal of the gas is likely to be connected to processes that affect the galaxies in other ways, such as tidal destruction (see e.g. paper XI) and morphological transformation (see Paper IX).

Acknowledgements. The European Southern Observatory and La Palma Observatory are acknowledged for assigning observing time for this project. We thank Roland den Hartog and Pascal de Theije who played an important rôle in the (early stages of the) imaging program, and Andrea Biviano for helpful comments on an earlier version of the manuscript. The observations for the imaging program on the Dutch telescope at La Silla were made by: Jasper Arts, Jos de Bruijne, Erik Brogt, Veerle Coupe, Peter van Dokkum, Ronnie Hoogerwerf, Magiel Janson, Dirk Janssens, Ciska Kemper, Jaron Kurk, Arjen van der Meer, Richard Naber, Celeste Ponsioen and Tom Voskes.

\section{References}

Andreon, S. 1998, ApJ, 501, 533

Biviano, A., Katgert, P., Mazure, A., et al. 1997, A\&A, 321, 84 (Paper III) 
Biviano, A., Katgert, P., Thomas, T., \& Adami, C. 2002, A\&A, 387, 8 (Paper XI)

Bower, R. G., Lucey, J. R., \& Ellis, R. S. 1992, MNRAS, 254, 601

Carlberg, R. G., Yee, H. K. C., \& Ellingson, E. 1997, ApJ, 485, L13

Couch, W. J., \& Sharples, R. M. 1987, MNRAS, 229, 423

Dale, D. A., Giovanelli, R., Haynes, M. P., Hardy, E., \& Campusano, L. E. 2001, AJ, 121, 1886

den Hartog, R., \& Katgert, P. 1996, MNRAS, 279, 349

de Vaucouleurs, G. 1959, in Handbuch der Physik, ed. S. Flügge

de Vaucouleurs, G. 1963, ApJS, 8, 31

de Theije, P. A. M., \& Katgert, P. 1999, A\&A, 341, 371 (Paper VI)

Dressler, A. 1980, ApJS, 42, 565

Dressler, A., \& Gunn, J. E. 1983, ApJ, 270, 7

Dressler, A., Oemler, A. Jr., Couch, W. J., et al. 1997, ApJ, 490, 577

Dressler, A., Smail, I., Poggianti, B. M., et al. 1999, ApJS, 122, 51

Ellingson, E., Lin, H., Yee, H. K. C., \& Carlberg, R. G. 2001, ApJ, 547, 609

Ellis, R. S., Smail, I., Dressler, A., et al. 1997, ApJ, 483, 582

Ellis, R. S., Abraham, R. G., \& Dickinson, M. 2001, ApJ, 551, 111

Fabricant, D., Franx, M., \& van Dokkum, P. G. 2000, ApJ, 539, 577

Fasano, G., Poggianti, B. M., Couch, W. J., et al. 2000, ApJ, 542, 673

Folkes, S. R., Lahav, O., \& Maddox, S. J. 1996, MNRAS, 283, 651

Gavazzi, G., Franzetti, P., Scodeggio, M., Boselli, A., \& Pierini D. 2000, A\&A, 361, 863

Hubble, E. 1926, ApJ, 64, 321

Hubble, E. 1936, The Realm of Nebulae. (New Haven: Yale Univ. Press)

Jones, L., Smail, I., \& Couch, W. J. 2000, ApJ, 528, 118

Katgert, P., Mazure, A., Perea, J., et al. 1996, A\&A, 310, 8 (Paper I)

Katgert, P., Mazure, A., \& den Hartog, R., et al. 1998, A\&AS, 129, 399 (Paper V)

Katgert, P., Biviano, A., \& Mazure, A. 2004, ApJ, 600, 657 (Paper XII)
Kelson, D. D., Illingworth, G. D., van Dokkum, P., \& Franx, M. 2000, ApJ, 531, 184

Kent, S. M. 1985, ApJS, 59, 115

Kuntschner, H., \& Davies, R. L. 1998, MNRAS, 295, L29

Lahav, O., Naim, A., Sodré, L. Jr., \& Storrie-Lombardi, M. C. 1996, MNRAS, 283, 207

Lucey, J. R., Guzman, R., Carter, D., \& Terlevich, R. J. 1991, MNRAS, 253, 584

Morgan, W. W. 1958, PASP, 70, 364

Naim, A., Lahav, O., Buta, R. J., et al. 1995a, MNRAS, 274, 1107

Naim, A., Lahav, O., Sodré, L. Jr., \& Storrie-Lombardi, M. C. 1995b, MNRAS, 275, 567

Peletier, R. F., Vazdekis, A., Arribas, S., et al. 1999, MNRAS, 310, 863

Poggianti, B., Smail, I., Dressler, A., et al. 1999, ApJ, 518, 576

Poggianti, B., Bridges, T. J., Komiyama, Y., et al. 2004, ApJ, 601, 197

Sandage, A. R. 1961, The Hubble Atlas of Galaxies, Carnegie Institute of Washington, Washington

Schechter, P. L., \& Dressler, A. 1987, AJ, 94, 563

Simien, F., \& de Vaucouleurs, G. 1986, ApJ, 302, 564

Smail, I., Kuntschner, H., Kodama, T., et al. 2001, MNRAS, 323, 839

Solanes, J. M., Manrique, A., Garcia-Gomez, C., et al. 2001, ApJ, 548, 97

Thomas, T., \& Katgert, P. 2006, A\&A, 446, 31 (Paper IX)

van den Bergh, S. 1960a, ApJ, 131, 215

van den Bergh, S. 1960b, ApJ, 131, 558

van den Bergh, S. 1976, ApJ, 206, 883

van den Bergh, S. 1997, AJ, 113, 2054

van Dokkum, P. G., \& Franx, M. 1996, MNRAS, 281, 985

van Dokkum, P. G., Franx, M., Kelson, D. D., \& Illingworth, G. D. 1998, ApJ, 504, L17

Zaritsky, D., Zabludoff, A. I., \& Willick, J. A. 1995, AJ, 110, 1602 